\title{
28 Research Square \\ Food Marketing, Eating Behaviors and Gender Among Children and Adolescents: A Scoping Review
}

\section{Luciana Castronuovo ( $\square$ luciana.castronuovo@ficargentina.org )}

Fundación Interamericana del Corazón Argentina. Arévalo $23641^{\circ}$ A CABA (1425) Argentina https://orcid.org/0000-0002-1883-6741

\section{Leila Guarnieri}

Other Press

\section{Victoria Tiscornia \\ Other Press}

\section{Lorena Allemandi}

Other Press

\section{Research}

Keywords: Food marketing, Gender perspective, Children and adolescents, Scoping review

Posted Date: January 11th, 2021

DOl: https://doi.org/10.21203/rs.3.rs-141456/v1

License: (c) (i) This work is licensed under a Creative Commons Attribution 4.0 International License. Read Full License 


\section{Abstract}

Background Pervasive marketing of unhealthy foods is one of the main drivers behind the global epidemic of childhood and adolescent overweight and obesity. Sex and gender differences come into play in the design of and responses to these marketing strategies, contributing to the perpetuation of stereotyped behavior and generating disparities in food choices and health among boys and girls. The purpose of this paper is to review the current the literature regarding gender differences in food marketing design and perception among children and adolescents to facilitate evidence-based policy dialogues to address genderbased health disparities in NCD prevention.

Methods Scoping review of articles published in scientific journals in English and Spanish from 2003 on that addressed the influence of food marketing on eating behaviors among children and adolescents including a gender perspective. The methodological quality of each article was assessed following criteria specific to each study design.

Results A total of 40 articles (42 studies) were included in the review. 19 were experimental and 23 had descriptive, cross-sectional designs. 22 studies were found to have low methodological quality, while 11 and 9 were of medium and high quality, respectively. 24 studies found gender-based differences with regards to the effect of food marketing on food intake, food choice and preferences, responses to specific marketing strategies and techniques, perceptions and attitudes towards food marketing and the need for its regulation, and advertising content and exposure.

Conclusions The evidence suggests that food marketing has a similar impact on the consumption of unhealthy foods on both boys and girls, but boys were found to be exposed to food advertising more intensively and their preferences to be more affected by this exposure, in line with a mostly male-dominant advertising content. Limitations of these studies include taking gender as an unproblematic construct equivalent to biological sex and the lack of studies focused on developing countries. As gender is a crosssectional dimension that interacts with other factors driving health disparities, an integrated gender perspective is needed in order to develop effective, evidence-based policies to control food marketing and tackle the childhood overweight pandemic.

\section{Background}

There is no doubt that the current childhood overweight epidemic is one of the most serious public health issues globally. The most recent global data suggest that $5.6 \%$ of all children under the age of five where overweight in 2019 , following an upward trend over the past 20 years[1], while in $2016,18 \%$ of the global population 5 to 18 years old suffered from the same condition[2]. Overweight causes not only many crippling physical illnesses, but also psychosocial issues, such as negative body image, reduced self-esteem, social discrimination/isolation and depression[3, 4], thus hindering the comprehensive development of children and adolescents.

One of the main culprits in this trend is the influence of food advertising on eating behaviors, particularly those that target children and promote the consumption of energy-dense, processed food products that are 
high in sugar, salt, and fats [5-9]. There is a growing body of evidence demonstrating that children's TV shows in Latin America include more food ads than programs targeting the general public; moreover, the food products marketed to children in TV have a lower nutritional quality than products advertised to other audiences [10]. This has also been documented in Argentina [11, 12]. The food industry invests heavily in marketing these products, most of which do not comply with current dietary guidelines, such as sugarcoated breakfast cereals, sweets and candy, soft drinks and fast food [13]. They also employ a wide range of media and diversity of strategies to deliver its messages, including advertising in school environments, food packaging, TV, internet and social media platforms $[10,14,15]$.

International recommendations to tackle the overweight epidemic $[7,13]$ include the restriction of food advertising, underscoring the urgent need to implement policies that limit, or even eliminate, the exposure of children and adolescents to marketing of food products with low nutritional quality.

Sex (biology) and gender (cultural) differences are relevant to overweight and obesity. Gender differences in food choices and dietary patterns are framed within social factors including societal expectations and stereotypes for males and females being transmitted via parental, peer and media influences. A literature search conducted by Sweeting[16] showed small male and female differences in obesity rates with no main predominance of either one within particular age groups. However, the study found differences in patterning of body fat, the fat levels at which health risks become apparent, levels of resting energy expenditure and energy requirements, ability to engage in certain physical activities and the consequences of obesity for the female reproductive system. Cultural differences identified in the study include food choices and dietary concerns, overall physical activity levels, body satisfaction and the long-term psychosocial consequences of childhood and adolescent obesity.

In this context, it is necessary to gain better understanding of the interplay of gender and food marketing, since there is evidence that health indicators, particularly those that are intimately related to lifestyle choices -eating behaviors, physical activity, etc.- are heavily determined by gender [17]. The evidence also shows that advertising tends to offer and exacerbate traditional and stereotyped images of men and women and gender roles[18-20]. Advertising often contributes to consolidate gender stereotypes that perpetuate culturally rooted social and health disparities and condition differential food choices among boys and girls.

Here we present a scoping review of literature dealing with food marketing aimed at children and adolescents that also include a gender perspective. This analysis was conducted in the framework of the multi-component regional research project "Food Marketing targeted to kids: a collaborative and policyoriented study in Argentina, Bolivia, Guatemala and Peru". The overall purpose of this review was to assess the current state of the evidence regarding gender differences in the influence, design and perception of food marketing and to identify knowledge domains that would benefit from further inquiry. The ultimate goal of this study was to facilitate evidence-based policy dialogues to reduce gender-based health disparities in NCD prevention policies.

\section{Methods}


We followed the guidelines proposed by Levac, Colquhoun and O'Malley [21] to conduct scoping reviews. We identified and assessed the methodological quality of published scientific articles that addressed the influence of food marketing on eating behaviors among children and adolescents including a gender perspective. Specific dimensions of interest were gender differences in: a) the effect of food marketing on food intake; $b$ ) the effect of food marketing in food choice and preferences; $c$ ) responses to specific marketing strategies and techniques; $d$ ) perceptions and attitudes towards food marketing and the need for its regulation; and e) advertising content and exposure.

\section{Inclusion criteria}

Studies were considered eligible for this scoping review if they met the following inclusion criteria:

a. presented evidence pertaining to children and adolescents up to 18 years old;

b. was published in Spanish or English from 2003 on,

c. addressed at least one of the following themes:

- a theoretical discussion of the association between food marketing strategies and gender disparities;

- a model to estimate how food advertising incorporates gender in combination with other attributes (age, race, socioeconomic level);

- differential food marketing strategies to target specific genders;

- an evaluation of gender-based differences in the influence of food marketing on eating behaviors;

The decision to include 18-year-olds as adolescents resulted in the incorporation of a few works that focus on young adults. Editorial and commentary pieces, "gray" literature and articles that did not report specific data pertaining children and adolescents were excluded from this study.

\section{Search strategy}

An electronic database search was conducted using PubMed and EBSCOHost; the latter includes Business Source Premier, CAB Abstracts, EconLit, LISTA, SocINDEX, Cairn.info, Directory of Open Access Journals (DOAJ), ERIC, HeinOnline, JSTOR, OECD iLibrary, Persée, SciELO, World Bank eLibrary, MEDLINE, EMBASE and LILACS. The structure of the search was [(food marketing AND gender) OR (food marketing AND femininity) OR (food marketing AND masculinity)] AND (child OR children OR teenager OR boy OR girl). Additional articles for potential inclusion were identified in a second stage by hand-searching the reference lists in relevant articles.

\section{Data extraction}

Two researchers conducted a two-stage screening process to identify articles that met all inclusion criteria. First, titles and abstracts were analyzed to exclude clearly irrelevant articles and remove duplicates, and then the eligibility of these pre-selected articles was confirmed by evaluating the full text. Disagreements regarding inclusion/exclusion were settled by discussion between the two researchers. 
The following information was collected for each included study: full reference (authors, year of publication, journal), country, study design, objectives and main results. The main themes and sub-themes explored in each paper were identified and a narrative synthesis was developed inductively based on these themes.

\section{Quality assessment}

The quality of each article was evaluated using different guidelines that establish the criteria to be met for each study design. Relevance, Appropriateness, Transparency, Soundness (RATS) guidelines (https://www.equator-network.org/reporting-guidelines/qualitative-research-review-guidelines-rats/) were used for qualitative studies, Effective Public Health Practice Project (EPHPP) guidelines, (https://merst.ca/wp-content/uploads/2018/02/quality-assessment-tool_2010.pdf) for experimental studies, and CEBMa for cross-sectional designs (https://www.cebma.org/wp-content/uploads/CriticalAppraisal-Questions-for-a-Cross-Sectional-Study-july-2014.pdf). Studies that analyzed advertising content were evaluated using an instrument specifically designed for this purpose, based on the methodology proposed by Leamy and colleagues [22].

The selected studies were classified according to the percentage of criteria met: low ( $<33 \%)$, medium (34$66 \%$ ), and high quality (>66\%).

\section{Results}

Electronic searches in PubMed and EBSCOHost yielded 364 potentially relevant articles, of which 120 were duplicates and were removed from the list. Screening of title and abstracts excluded 144 articles, leaving a total of 100 potentially relevant articles of which 62 were excluded after full text assessment. The remaining

38 articles were included in this review. By analyzing the reference lists in these articles, an additional 2 were identified as relevant for this work, resulting in 40 articles selected for the review (Fig. 1). A total of 42 studies (Table 1) were analyzed -two articles included two separate studies each- of which 19 were experimental and 23 had descriptive, cross-sectional designs. Over half the studies (n 22) were found to have low methodological quality, while 11 and 9 were of medium and high quality, respectively (Table 2). 
Table 1

Studies included in the review. Summary and main findings.

\begin{tabular}{|c|c|c|c|c|c|}
\hline Reference & Country & Study design & $\begin{array}{l}\text { Quality } \\
\text { score }\end{array}$ & Objective & Main outcomes \\
\hline $\begin{array}{l}\text { Ueda et al., } \\
2012 \text { [23] }\end{array}$ & India & Experimental & LOW & $\begin{array}{l}\text { To evaluate } \\
\text { exposure to } \\
\text { advertising of } \\
\text { less healthy } \\
\text { food and its } \\
\text { association } \\
\text { with eating } \\
\text { behaviors and } \\
\text { BMl among } \\
\text { children (3- } \\
13 \text { years old). }\end{array}$ & $\begin{array}{l}\text { Brand logo recognition: } \\
30 \text { to } 80 \% \text {. Capacity for } \\
\text { logo recognition } \\
\text { increased with age and } \\
\text { socio-economic status. } \\
\text { Adjusting by these } \\
\text { variables and gender, } \\
\text { logo recognition was } \\
\text { associated to higher } \\
\text { BMI and nutritional } \\
\text { knowledge, but not } \\
\text { with preferences } \\
\text { towards less healthy } \\
\text { foods or purchase } \\
\text { requests. No significant } \\
\text { differences were found } \\
\text { between genders. }\end{array}$ \\
\hline $\begin{array}{l}\text { Chernin, } 2008 \\
\text { [24] }\end{array}$ & USA & Experimental & MEDIUM & $\begin{array}{l}\text { To examine the } \\
\text { influence of } \\
\text { two } \\
\text { advertisements } \\
\text { (breakfast } \\
\text { cereal, juice } \\
\text { powder) on } \\
\text { food product } \\
\text { preferences } \\
\text { among children } \\
\text { ( } 5-10 \text { years } \\
\text { old). }\end{array}$ & $\begin{array}{l}\text { Exposure to food } \\
\text { advertising increased } \\
\text { children's preference } \\
\text { for the advertised } \\
\text { products. This } \\
\text { influence was stronger } \\
\text { among boys than } \\
\text { among girls, although } \\
\text { both genders were } \\
\text { depicted in the } \\
\text { advertisements. }\end{array}$ \\
\hline $\begin{array}{l}\text { Tarabashkina } \\
\text { et al., } \\
2016[25]\end{array}$ & Australia & Experimental & MEDIUM & $\begin{array}{l}\text { To assess the } \\
\text { role of product } \\
\text { evaluations, } \\
\text { nutritional } \\
\text { knowledge and } \\
\text { awareness of } \\
\text { persuasive } \\
\text { intent on food } \\
\text { choices among } \\
\text { children and } \\
\text { adolescents } \\
\text { (7-13 years } \\
\text { old). }\end{array}$ & $\begin{array}{l}\text { When participants } \\
\text { showed little nutritional } \\
\text { knowledge and low } \\
\text { awareness of } \\
\text { persuasive intent } \\
\text { behind advertising and } \\
\text { believed the advertised } \\
\text { product to be healthy, } \\
\text { they were more likely to } \\
\text { choose the advertised } \\
\text { product. No significant } \\
\text { differences were found } \\
\text { by gender and age in } \\
\text { the control and } \\
\text { experimental groups. }\end{array}$ \\
\hline
\end{tabular}




\begin{tabular}{|c|c|c|c|c|c|}
\hline Reference & Country & Study design & $\begin{array}{l}\text { Quality } \\
\text { score }\end{array}$ & Objective & Main outcomes \\
\hline $\begin{array}{l}\text { Norman et } \\
\text { al., } 2018 \text { [26] }\end{array}$ & Australia & Experimental & MEDIUM & $\begin{array}{l}\text { To evaluate the } \\
\text { impact of } \\
\text { advertising (TV } \\
\text { and online } \\
\text { gaming } \\
\text { platforms) on } \\
\text { the amount of } \\
\text { food consumed } \\
\text { among children } \\
\text { and } \\
\text { adolescents } \\
\text { (7-12 years } \\
\text { old). }\end{array}$ & $\begin{array}{l}\text { Children exposed to } \\
\text { food ads in TV and } \\
\text { online gaming } \\
\text { platforms ate more } \\
\text { food while snacking, } \\
\text { compared to the group } \\
\text { exposed only to non- } \\
\text { food ads in TV. There } \\
\text { were no significant } \\
\text { differences or } \\
\text { interactions by age, } \\
\text { gender, brand } \\
\text { recognition, and } \\
\text { household weekly } \\
\text { income. }\end{array}$ \\
\hline $\begin{array}{l}\text { Anderson et } \\
\text { al., 2015[27] }\end{array}$ & Canada & Experimental & LOW & $\begin{array}{l}\text { To evaluate the } \\
\text { influence of } \\
\text { food ads in TV } \\
\text { on the energy } \\
\text { intake of } \\
\text { children ( } 9- \\
14 \text { years old) } \\
\text { with normal } \\
\text { and excess } \\
\text { weight. }\end{array}$ & $\begin{array}{l}\text { Girls with excess } \\
\text { weight showed a } \\
\text { higher increase of their } \\
\text { energy intake } \\
\text { compared to girls with } \\
\text { normal weight and } \\
\text { boys, suggesting } \\
\text { higher vulnerability to } \\
\text { food advertising. Girls } \\
\text { exposed to food ads } \\
\text { were more likely to find } \\
\text { the TV show } \\
\text { acceptable, compared } \\
\text { to girls that viewed } \\
\text { non-food ads. Among } \\
\text { boys, TV show } \\
\text { acceptability was not } \\
\text { influenced by the } \\
\text { product type } \\
\text { advertised. }\end{array}$ \\
\hline $\begin{array}{l}\text { Velazquez } \\
\text { and Pasch, } \\
2014[28]\end{array}$ & USA & Experimental & LOW & $\begin{array}{l}\text { To assess the } \\
\text { relationship } \\
\text { between } \\
\text { attention to } \\
\text { unhealthy food } \\
\text { ads and food } \\
\text { preferences } \\
\text { and choices } \\
\text { among children } \\
\text { and } \\
\text { adolescents } \\
\text { (8-15 years } \\
\text { old). }\end{array}$ & $\begin{array}{l}\text { The amount of time } \\
\text { and frequency of } \\
\text { exposure to unhealthy } \\
\text { foods was significantly } \\
\text { associated to } \\
\text { preferences for } \\
\text { unhealthy foods. This } \\
\text { association was not } \\
\text { affected by sex, age or } \\
\text { BMI. }\end{array}$ \\
\hline
\end{tabular}




\begin{tabular}{|c|c|c|c|c|c|}
\hline Reference & Country & Study design & $\begin{array}{l}\text { Quality } \\
\text { score }\end{array}$ & Objective & Main outcomes \\
\hline \multirow{3}{*}{$\begin{array}{l}\text { Keller et } \\
\text { al.,2012[29] }\end{array}$} & \multirow[t]{3}{*}{ USA } & Study 1: & LOW & \multirow{2}{*}{$\begin{array}{l}\text { To determine if } \\
\text { the presence of } \\
\text { a familiar } \\
\text { brand affects } \\
\text { test-meal intake } \\
\text { among young } \\
\text { children (4- } \\
6 \text { years old). }\end{array}$} & \multirow[b]{2}{*}{$\begin{array}{l}\text { Energy intake } \\
\text { increased by } 41 \mathrm{kcal} \\
\text { in children with excess } \\
\text { weight when presented } \\
\text { with branded food } \\
\text { items than when } \\
\text { offered unbranded } \\
\text { foods (i.e. in unmarked, } \\
\text { plain white containers). } \\
\text { In contrast, children } \\
\text { with normal weight } \\
\text { consumed } 45 \mathrm{kcal} \\
\text { less when presented } \\
\text { with branded foods, } \\
\text { compared to the } \\
\text { unbranded condition. } \\
\text { There was no } \\
\text { significant difference } \\
\text { between boys and girls. }\end{array}$} \\
\hline & & Experimental & & & \\
\hline & & $\begin{array}{l}\text { Study 2: } \\
\text { Experimental }\end{array}$ & LOW & $\begin{array}{l}\text { To determine if } \\
\text { the presence of } \\
\text { a familiar fast- } \\
\text { food brand } \\
\text { affects test- } \\
\text { meal intake } \\
\text { among children } \\
\text { (7-9 years old). }\end{array}$ & $\begin{array}{l}\text { The boys' energy intake } \\
\text { was similar in the } \\
\text { presence of fast-food } \\
\text { branded/unbranded } \\
\text { food items. In turn, girls } \\
\text { consumed } \sim 100 \mathrm{kcal} \\
\text { more when the items } \\
\text { were accompanied by } \\
\text { a fast-food brand. }\end{array}$ \\
\hline $\begin{array}{l}\text { Anschutz et } \\
\text { al., 2009[30] }\end{array}$ & Netherlands & Experimental & LOW & $\begin{array}{l}\text { To evaluate the } \\
\text { effects of TV } \\
\text { food ads on } \\
\text { concurrent non- } \\
\text { advertised } \\
\text { sweet snack } \\
\text { food intake in } \\
\text { young children } \\
\text { ( } 8-12 \text { years } \\
\text { old). }\end{array}$ & $\begin{array}{l}\text { Boys who viewed food } \\
\text { ads presented higher } \\
\text { snack intake than boys } \\
\text { exposed to neutral } \\
\text { (non-food) ads. In turn, } \\
\text { snack intake was } \\
\text { slightly lower among } \\
\text { girls when they viewed } \\
\text { food ads than when } \\
\text { the viewed neutral ads. }\end{array}$ \\
\hline
\end{tabular}




\begin{tabular}{|c|c|c|c|c|c|}
\hline Reference & Country & Study design & $\begin{array}{l}\text { Quality } \\
\text { score }\end{array}$ & Objective & Main outcomes \\
\hline $\begin{array}{l}\text { Dixon et al., } \\
2014[31]\end{array}$ & Australia & Experimental & LOW & $\begin{array}{l}\text { To evaluate } \\
\text { responses to } \\
\text { promotional } \\
\text { elements - } \\
\text { nutrient content } \\
\text { claims, sports } \\
\text { celebrity } \\
\text { endorsements } \\
\text { and premium } \\
\text { offers- in food } \\
\text { packaging } \\
\text { among pre- } \\
\text { adolescent } \\
\text { children } \\
\text { (average: } \\
11 \text { years old). }\end{array}$ & $\begin{array}{l}\text { Children were more } \\
\text { likely to choose energy- } \\
\text { dense, nutrient-poor } \\
\text { food products when } \\
\text { their packs showed } \\
\text { nutrient claims (both } \\
\text { genders) or sports } \\
\text { celebrities (only boys), } \\
\text { compared to control } \\
\text { conditions (no } \\
\text { promotions). }\end{array}$ \\
\hline $\begin{array}{l}\text { Hobin et al., } \\
2012[32]\end{array}$ & Canada & Experimental & LOW & $\begin{array}{l}\text { To determine if } \\
\text { children make } \\
\text { healthier food } \\
\text { choices if toy } \\
\text { premiums are } \\
\text { only offered } \\
\text { with healthier } \\
\text { fast-food meals } \\
\text { (ages 6- } \\
12 \text { years old). }\end{array}$ & $\begin{array}{l}\text { Children were more } \\
\text { likely to choose } \\
\text { healthier meals when } \\
\text { toy premiums were } \\
\text { only offered with meals } \\
\text { that met nutritional } \\
\text { criteria. This effect was } \\
\text { stronger among boys } \\
\text { than among girls. }\end{array}$ \\
\hline $\begin{array}{l}\text { Ogle et al., } \\
2017[33]\end{array}$ & USA & Experimental & LOW & $\begin{array}{l}\text { To determine if } \\
\text { adding licensed } \\
\text { cartoon } \\
\text { characters to } \\
\text { healthy } \\
\text { food/beverages } \\
\text { packaging can } \\
\text { increase } \\
\text { attention to and } \\
\text { preference for } \\
\text { these products } \\
\text { among children } \\
\text { (6-9 years old). }\end{array}$ & $\begin{array}{l}\text { Children paid more } \\
\text { attention to products } \\
\text { with characters and } \\
\text { preferred less-healthy } \\
\text { products, although they } \\
\text { preferred products } \\
\text { without characters over } \\
60 \% \text { of the time. Age, } \\
\text { sex, and the specific } \\
\text { cartoon character were } \\
\text { significant influences } \\
\text { on product choice, with } \\
\text { characters being } \\
\text { preferred by younger } \\
\text { boys. }\end{array}$ \\
\hline
\end{tabular}




\begin{tabular}{|c|c|c|c|c|c|}
\hline Reference & Country & Study design & $\begin{array}{l}\text { Quality } \\
\text { score }\end{array}$ & Objective & Main outcomes \\
\hline $\begin{array}{l}\text { Wonderlich- } \\
\text { Tierney et } \\
\text { al.,2013[34] }\end{array}$ & USA & Experimental & LOW & $\begin{array}{l}\text { To examine the } \\
\text { impact of TV } \\
\text { ads on food } \\
\text { intake by sex } \\
\text { and } \\
\text { transportability } \\
\text {-"the tendency } \\
\text { to become } \\
\text { absorbed in a } \\
\text { narrative"- } \\
\text { among } \\
\text { adolescents } \\
\text { and young } \\
\text { adults } \\
\text { (average: } \\
19 \text { years old). }\end{array}$ & $\begin{array}{l}\text { Participants with high } \\
\text { transportability } \\
\text { presented higher food } \\
\text { intake when viewing } \\
\text { food ads vs. neutral } \\
\text { ads. Women with high } \\
\text { transportability ate } \\
\text { more than women with } \\
\text { low transportability, } \\
\text { regardless of the type } \\
\text { of ad. The study did } \\
\text { not find any significant } \\
\text { main effects of ad } \\
\text { type, sex or } \\
\text { transportability on food } \\
\text { intake. }\end{array}$ \\
\hline $\begin{array}{l}\text { Anschutz et } \\
\text { al., 2011[35] }\end{array}$ & Netherlands & Experimental & LOW & $\begin{array}{l}\text { To evaluate } \\
\text { direct effects of } \\
\text { viewing TV } \\
\text { food ads on the } \\
\text { concurrent } \\
\text { intake of non- } \\
\text { advertised } \\
\text { snack foods } \\
\text { and possible } \\
\text { differences by } \\
\text { sex among } \\
\text { young adults } \\
\text { (average: } \\
20 \text { years old) }\end{array}$ & $\begin{array}{l}\text { Snack intake among } \\
\text { women increased when } \\
\text { viewing food ads (vs. } \\
\text { non-food ads). The } \\
\text { opposite pattern was } \\
\text { found for men, who } \\
\text { decreased their food } \\
\text { intake when viewing } \\
\text { food ads. }\end{array}$ \\
\hline $\begin{array}{l}\text { Adams and } \\
\text { Geuens, } \\
2007[36]\end{array}$ & Belgium & Experimental & LOW & $\begin{array}{l}\text { To examine } \\
\text { responses to } \\
\text { healthy and } \\
\text { unhealthy } \\
\text { slogans in ads } \\
\text { for food } \\
\text { products } \\
\text { perceived as } \\
\text { healthy or } \\
\text { unhealthy } \\
\text { among } \\
\text { adolescents } \\
\text { (15 years old). }\end{array}$ & $\begin{array}{l}\text { Although no significant } \\
\text { main effects were } \\
\text { found for the } \\
\text { healthfulness of the } \\
\text { slogan or the product, } \\
\text { there was a significant } \\
\text { interaction effect. More } \\
\text { significantly positive } \\
\text { responses and } \\
\text { increased purchase } \\
\text { intent were elicited } \\
\text { when the healthfulness } \\
\text { of the slogan and the } \\
\text { perceived } \\
\text { healthfulness of the } \\
\text { product were } \\
\text { consistent. } \\
\text { Adolescents that were } \\
\text { highly concerned about } \\
\text { health responded more } \\
\text { positively towards } \\
\text { healthy slogans. No } \\
\text { significant differences } \\
\text { were found between } \\
\text { genders. }\end{array}$ \\
\hline
\end{tabular}




\begin{tabular}{|c|c|c|c|c|c|}
\hline Reference & Country & Study design & $\begin{array}{l}\text { Quality } \\
\text { score }\end{array}$ & Objective & Main outcomes \\
\hline $\begin{array}{l}\text { Harris et al., } \\
2018[37]\end{array}$ & USA & Experimental & LOW & $\begin{array}{l}\text { To evaluate the } \\
\text { effects of } \\
\text { health } \\
\text { messages in } \\
\text { child-directed } \\
\text { advertising for } \\
\text { unhealthy food } \\
\text { products (7- } \\
11 \text { years old). }\end{array}$ & $\begin{array}{l}\text { Children's perception of } \\
\text { healthfulness of } \\
\text { unhealthy products } \\
\text { increased when } \\
\text { associated to nutrition } \\
\text { and/or physical activity } \\
\text { messages. No } \\
\text { significant differences } \\
\text { were found between } \\
\text { genders. }\end{array}$ \\
\hline $\begin{array}{l}\text { Gines } \\
\text { Geraldo and } \\
\text { Machado } \\
\text { Pinto e Silva, } \\
\text { 2012[38] }\end{array}$ & Brazil & Experimental & LOW & $\begin{array}{l}\text { To describe } \\
\text { visual memory } \\
\text { of the } \\
\text { packaging of } \\
\text { snacks and } \\
\text { filled cookies in } \\
\text { relation to } \\
\text { nutritional } \\
\text { status, school } \\
\text { grade and } \\
\text { gender in } \\
\text { school children } \\
\text { (6-10 years } \\
\text { old). }\end{array}$ & $\begin{array}{l}\text { Both genders exhibited } \\
\text { similar memory levels } \\
\text { for colors depicted in } \\
\text { the packaging of both } \\
\text { products. Girls } \\
\text { remembered imagery } \\
\text { and characters } \\
\text { depicted on the } \\
\text { packaging better than } \\
\text { boys. Nutritional status } \\
\text { was not a significant } \\
\text { influence on visual } \\
\text { memory of pack } \\
\text { features. }\end{array}$ \\
\hline $\begin{array}{l}\text { Anschutz et } \\
\text { al., 2010[39] }\end{array}$ & Netherlands & Experimental & LOW & $\begin{array}{l}\text { To assess the } \\
\text { effect of } \\
\text { viewing adult- } \\
\text { directed } \\
\text { advertising } \\
\text { (energy-dense, } \\
\text { "light" products, } \\
\text { and non-food } \\
\text { products) on } \\
\text { concurrent } \\
\text { snack intake in } \\
\text { children (8- } \\
12 \text { years old) } \\
\text { and possible } \\
\text { moderating } \\
\text { effects of } \\
\text { maternal } \\
\text { behaviors. }\end{array}$ & $\begin{array}{l}\text { Food intake decreased } \\
\text { significantly with age } \\
\text { and increased with } \\
\text { hunger and liking of } \\
\text { the test food products. } \\
\text { Children who received } \\
\text { maternal } \\
\text { encouragement to be } \\
\text { thin ate more when } \\
\text { exposed to food ads } \\
\text { (vs. non-food ads), } \\
\text { while children that did } \\
\text { not receive such } \\
\text { encouragement ate } \\
\text { more when exposed to } \\
\text { non-food ads. No } \\
\text { significant main effect } \\
\text { was found for ad types } \\
\text { (energy-dense food, } \\
\text { "light" food, non-food) } \\
\text { or gender. }\end{array}$ \\
\hline
\end{tabular}




\begin{tabular}{|c|c|c|c|c|c|}
\hline Reference & Country & Study design & $\begin{array}{l}\text { Quality } \\
\text { score }\end{array}$ & Objective & Main outcomes \\
\hline \multirow[t]{2}{*}{$\begin{array}{l}\text { Castonguay } \\
\text { and Bakir, } \\
2018[17]\end{array}$} & USA & Experimental & LOW & $\begin{array}{l}\text { To analyze } \\
\text { gender } \\
\text { differences in } \\
\text { nutritional } \\
\text { understanding, } \\
\text { intentions to } \\
\text { engage in } \\
\text { physical } \\
\text { activity and } \\
\text { responses to an } \\
\text { advertisement } \\
\text { for a unhealthy } \\
\text { food product } \\
\text { with and } \\
\text { without } \\
\text { imagery of } \\
\text { children } \\
\text { practicing } \\
\text { sports, in } \\
\text { children } 5 \text { to } \\
11 \text { years old. }\end{array}$ & $\begin{array}{l}\text { Compared to girls, boys } \\
\text { that viewed an ad } \\
\text { portraying physical } \\
\text { activity were more } \\
\text { likely to believe that } \\
\text { eating the advertised } \\
\text { food (sugar-coated } \\
\text { cereal) would make } \\
\text { them stronger, } \\
\text { compared to girls and } \\
\text { to boys who viewed an } \\
\text { ad without such } \\
\text { images. }\end{array}$ \\
\hline & & $\begin{array}{l}\text { Descriptive, } \\
\text { cross- }^{-} \\
\text {sectional. } \\
\text { Content } \\
\text { analysis }\end{array}$ & $\mathrm{HIGH}$ & $\begin{array}{l}\text { To analyze the } \\
\text { content of food } \\
\text { ads aired } \\
\text { during children- } \\
\text { directed TV } \\
\text { shows. }\end{array}$ & $\begin{array}{l}\text { Most ads shown during } \\
\text { children-directed TV } \\
\text { shows exclusively male } \\
\text { characters, while } 18.8 \% \\
\text { of ads featured female } \\
\text { characters exclusively, } \\
\text { and } 6.7 \% \text { featured } \\
\text { males and females. } \\
\text { Healthy food products } \\
\text { were associated to } \\
\text { girls, while healthy } \\
\text { activities were } \\
\text { predominantly } \\
\text { associated to boys. }\end{array}$ \\
\hline $\begin{array}{l}\text { Childs and } \\
\text { Maher, } \\
\text { 2003[40] }\end{array}$ & USA & $\begin{array}{l}\text { Descriptive, } \\
\text { cross- } \\
\text { sectional. } \\
\text { Content } \\
\text { analysis }\end{array}$ & $\mathrm{HIGH}$ & $\begin{array}{l}\text { To examine the } \\
\text { use of gender } \\
\text { in children- } \\
\text { directed TV } \\
\text { food ads. }\end{array}$ & $\begin{array}{l}\text { There was } \\
\text { overrepresentation of } \\
\text { male voices and } \\
\text { characters portrayed in } \\
\text { the ads, indicating that } \\
\text { a gender bias towards } \\
\text { male audiences exists } \\
\text { in food advertising to } \\
\text { children. }\end{array}$ \\
\hline
\end{tabular}




\begin{tabular}{|c|c|c|c|c|c|}
\hline Reference & Country & Study design & $\begin{array}{l}\text { Quality } \\
\text { score }\end{array}$ & Objective & Main outcomes \\
\hline $\begin{array}{l}\text { Skatrud- } \\
\text { Mickelson et } \\
\text { al., 2012[41] }\end{array}$ & USA & $\begin{array}{l}\text { Descriptive, } \\
\text { cross- } \\
\text { sectional. } \\
\text { Content } \\
\text { analysis }\end{array}$ & $\mathrm{HIGH}$ & $\begin{array}{l}\text { To estimate } \\
\text { exposure of } \\
\text { children to food } \\
\text { brand } \\
\text { impressions in } \\
\text { top-grossing } \\
\text { movies. }\end{array}$ & $\begin{array}{l}\text { Exposure to food brand } \\
\text { impressions varied } \\
\text { among boys and girls } \\
\text { depending on age and } \\
\text { movie MPAA ratings. } \\
\text { Girls were more } \\
\text { exposed to brand } \\
\text { impressions from } \\
\text { G/PG-rated movies, } \\
\text { while boys were more } \\
\text { exposed from PG-13/R- } \\
\text { rated movies. }\end{array}$ \\
\hline $\begin{array}{l}\text { Harrison, } \\
2006[42]\end{array}$ & USA & $\begin{array}{l}\text { Descriptive, } \\
\text { cross- } \\
\text { sectional. } \\
\text { Content } \\
\text { analysis }\end{array}$ & $\mathrm{HIGH}$ & $\begin{array}{l}\text { To analyze } \\
\text { food } \\
\text { advertising } \\
\text { content in } \\
\text { children- } \\
\text { directed TV, } \\
\text { comparing ads } \\
\text { portraying } \\
\text { Black and non- } \\
\text { Black } \\
\text { characters. }\end{array}$ & $\begin{array}{l}\text { Male characters were } \\
\text { overrepresented in both } \\
\text { the ads that featured } \\
\text { Black characters and } \\
\text { those who did not. }\end{array}$ \\
\hline $\begin{array}{l}\text { Olivares et } \\
\text { al.,2011[43] }\end{array}$ & Chile & $\begin{array}{l}\text { Quantitative. } \\
\text { Descriptive, } \\
\text { cross- } \\
\text { sectional. }\end{array}$ & MEDIUM & $\begin{array}{l}\text { To explore } \\
\text { attitudes } \\
\text { towards food } \\
\text { ads among } \\
\text { school children. }\end{array}$ & $\begin{array}{l}65 \% \text { of participants } \\
\text { expressed a liking for } \\
\text { trying new foods and } \\
\text { beverages promoted in } \\
\text { TV ads. No significant } \\
\text { differences were found } \\
\text { between genders. }\end{array}$ \\
\hline $\begin{array}{l}\text { Cornwell et } \\
\text { al., 2014[44] }\end{array}$ & USA & $\begin{array}{l}\text { Quantitative. } \\
\text { Descriptive, } \\
\text { cross- } \\
\text { sectional. }\end{array}$ & MEDIUM & $\begin{array}{l}\text { To evaluate } \\
\text { associations } \\
\text { between BMI } \\
\text { and knowledge } \\
\text { of brands of } \\
\text { food products } \\
\text { high in fats, } \\
\text { salt and sugar } \\
\text { among } \\
\text { children, }\end{array}$ & $\begin{array}{l}\text { Knowledge of } \\
\text { packaged and fast } \\
\text { food brands was found } \\
\text { to be a predictive factor } \\
\text { of BMI among children. } \\
\text { No significant } \\
\text { differences were found } \\
\text { by age and gender, nor } \\
\text { according to hours } \\
\text { spent watching TV. }\end{array}$ \\
\hline $\begin{array}{l}\text { Klepp et al., } \\
2007[45]\end{array}$ & $\begin{array}{l}\text { Austria, } \\
\text { lceland, } \\
\text { Portugal, } \\
\text { Norway, } \\
\text { Belgium, } \\
\text { Denmark, } \\
\text { Spain, } \\
\text { Netherlands, } \\
\text { Sweden }\end{array}$ & $\begin{array}{l}\text { Quantitative. } \\
\text { Descriptive, } \\
\text { cross- } \\
\text { sectional. }\end{array}$ & $\mathrm{HIGH}$ & $\begin{array}{l}\text { To investigate } \\
\text { associations } \\
\text { between } \\
\text { exposure to } \\
\text { food ads in TV } \\
\text { and reported } \\
\text { fruit and } \\
\text { vegetable } \\
\text { intake among } \\
\text { children from } \\
\text { nine European } \\
\text { countries. }\end{array}$ & $\begin{array}{l}\text { Most children reported } \\
\text { higher exposure to ads } \\
\text { for unhealthy food } \\
\text { than for fruit and } \\
\text { vegetables, but boys } \\
\text { reported spending } \\
\text { slightly more time } \\
\text { watching TV than girls. } \\
\text { Exposure to ads for } \\
\text { healthy foods was } \\
\text { positively associated } \\
\text { with reported fruit and } \\
\text { vegetable intake. }\end{array}$ \\
\hline
\end{tabular}




\begin{tabular}{|c|c|c|c|c|c|}
\hline Reference & Country & Study design & $\begin{array}{l}\text { Quality } \\
\text { score }\end{array}$ & Objective & Main outcomes \\
\hline $\begin{array}{l}\text { Baldwin et } \\
\text { al., 2018[46] }\end{array}$ & Australia & $\begin{array}{l}\text { Quantitative. } \\
\text { Descriptive, } \\
\text { cross- } \\
\text { sectional. }\end{array}$ & LOW & $\begin{array}{l}\text { To examine } \\
\text { associations } \\
\text { between } \\
\text { internet and } \\
\text { social media } \\
\text { behavior and } \\
\text { unhealthy food } \\
\text { intake among } \\
\text { children and } \\
\text { adolescents } \\
\text { (10-16 years } \\
\text { old). }\end{array}$ & $\begin{array}{l}\text { Exposure to advertising } \\
\text { was associated with a } \\
\text { higher intake of the } \\
\text { advertised products. } \\
\text { No significant } \\
\text { differences were found } \\
\text { between genders. }\end{array}$ \\
\hline $\begin{array}{l}\text { Bhawra et } \\
\text { al.,2018 [47] }\end{array}$ & Canada & $\begin{array}{l}\text { Quantitative. } \\
\text { Descriptive, } \\
\text { cross- } \\
\text { sectional. }\end{array}$ & MEDIUM & $\begin{array}{l}\text { To assess } \\
\text { support to food } \\
\text { policies among } \\
\text { youth in } \\
\text { Canada. }\end{array}$ & $\begin{array}{l}\text { Young women } \\
\text { expressed stronger } \\
\text { support than men for } \\
\text { nutrition symbols and } \\
\text { warnings school } \\
\text { policies, zoning } \\
\text { restrictions on } \\
\text { advertising, marketing } \\
\text { bans and maximum } \\
\text { salt limits. }\end{array}$ \\
\hline $\begin{array}{l}\text { Kumar et al., } \\
2015 \text { [48] }\end{array}$ & USA & $\begin{array}{l}\text { Quantitative. } \\
\text { Descriptive, } \\
\text { cross- } \\
\text { sectional. }\end{array}$ & MEDIUM & $\begin{array}{l}\text { To analyze } \\
\text { exposure to ads } \\
\text { for sugar- } \\
\text { sweetened } \\
\text { beverages } \\
\text { among } \\
\text { adolescents } \\
\text { (12-17 years } \\
\text { old). }\end{array}$ & $\begin{array}{l}\text { Between } 42 \% \text { and } 54 \% \\
\text { of the participants } \\
\text { reported exposure to } \\
\text { these advertisements } \\
\text { more than once a day. } \\
\text { Significant differences } \\
\text { were found by age, } \\
\text { ethnic group and } \\
\text { parents' educational } \\
\text { level, as well as gender. } \\
\text { Boys reported more } \\
\text { frequent exposure to } \\
\text { sugary sport beverage } \\
\text { ads than girls. }\end{array}$ \\
\hline $\begin{array}{l}\text { Adachi-Mejia } \\
\text { et al., } 2011 \\
\text { [49] }\end{array}$ & USA & $\begin{array}{l}\text { Quantitative. } \\
\text { Descriptive, } \\
\text { cross- } \\
\text { sectional. }\end{array}$ & MEDIUM & $\begin{array}{l}\text { To explore the } \\
\text { relationship } \\
\text { between weight } \\
\text { status and } \\
\text { receptivity to } \\
\text { food } \\
\text { advertising } \\
\text { among } \\
\text { adolescents. }\end{array}$ & $\begin{array}{l}\text { Having a favorite ad } \\
\text { was the chosen } \\
\text { indicator of receptivity. } \\
\text { Boys who reported } \\
\text { having a favorite ad } \\
\text { were more likely to } \\
\text { mention an ad for beer, } \\
\text { while girls were more } \\
\text { likely to mention an ad } \\
\text { for candy/sweets as } \\
\text { their favorite. }\end{array}$ \\
\hline
\end{tabular}




\begin{tabular}{|c|c|c|c|c|c|}
\hline Reference & Country & Study design & $\begin{array}{l}\text { Quality } \\
\text { score }\end{array}$ & Objective & Main outcomes \\
\hline $\begin{array}{l}\text { Buijzen et al., } \\
2008 \text { [50] }\end{array}$ & Netherlands & $\begin{array}{l}\text { Quantitative. } \\
\text { Descriptive, } \\
\text { cross- } \\
\text { sectional. }\end{array}$ & LOW & $\begin{array}{l}\text { To analyze } \\
\text { associations } \\
\text { between } \\
\text { exposure to } \\
\text { food } \\
\text { advertising and } \\
\text { consumption of } \\
\text { advertised } \\
\text { brands, } \\
\text { advertised } \\
\text { energy-dense } \\
\text { food product } \\
\text { categories and } \\
\text { food products } \\
\text { in general in } \\
\text { children (4- } \\
12 \text { years old). }\end{array}$ & $\begin{array}{l}\text { Exposure to food } \\
\text { advertising was } \\
\text { significantly } \\
\text { associated to } \\
\text { consumption of } \\
\text { advertised brands and } \\
\text { energy-dense food } \\
\text { product categories. } \\
\text { Intra-family } \\
\text { consumption-related } \\
\text { communication was } \\
\text { found to moderate the } \\
\text { relations between } \\
\text { advertising and food } \\
\text { consumption. No } \\
\text { significant differences } \\
\text { were found by age, } \\
\text { gender, and time spent } \\
\text { watching TV. }\end{array}$ \\
\hline $\begin{array}{l}\text { Grunseit et al, } \\
2012 \text { [51] }\end{array}$ & Australia & $\begin{array}{l}\text { Quantitative. } \\
\text { Descriptive, } \\
\text { cross- } \\
\text { sectional. }\end{array}$ & LOW & $\begin{array}{l}\text { To examine a) } \\
\text { opinions } \\
\text { regarding the } \\
\text { role of athletes } \\
\text { in the } \\
\text { promotion of } \\
\text { physical } \\
\text { activity and } \\
\text { obesity } \\
\text { prevention, b) } \\
\text { attitudes } \\
\text { towards the } \\
\text { promotion of } \\
\text { unhealthy food } \\
\text { products in } \\
\text { sports and c) } \\
\text { health-related } \\
\text { behaviors } \\
\text { among young } \\
\text { Australian } \\
\text { athletes. }\end{array}$ & $\begin{array}{l}\text { Most participants } \\
\text { agreed that athletes } \\
\text { should be positive role } \\
\text { models for active } \\
\text { lifestyles. Women } \\
\text { tended to agree more } \\
\text { with the proposition } \\
\text { than men. There were } \\
\text { also significant } \\
\text { differences in the } \\
\text { percentages of women } \\
\text { that were opposed to } \\
\text { advertising of alcohol } \\
\text { and unhealthy food in } \\
\text { sports and } \\
\text { endorsement by } \\
\text { professional athletes, } \\
\text { compared to men. }\end{array}$ \\
\hline $\begin{array}{l}\text { Olivares- } \\
\text { Cortes et al., } \\
2017 \text { [52] }\end{array}$ & Chile & $\begin{array}{l}\text { Quantitative. } \\
\text { Descriptive, } \\
\text { cross- } \\
\text { sectional. }\end{array}$ & $\mathrm{HIGH}$ & $\begin{array}{l}\text { To explore } \\
\text { attitudes and } \\
\text { opinions about } \\
\text { a new front-of- } \\
\text { pack nutrition } \\
\text { labeling system } \\
\text { among school- } \\
\text { children with } \\
\text { diverse } \\
\text { nutritional } \\
\text { status and } \\
\text { socioeconomic } \\
\text { level (8- } \\
12 \text { years old). }\end{array}$ & $\begin{array}{l}\text { Most children reported } \\
\text { liking to be informed } \\
\text { about the nutritional } \\
\text { value of food products } \\
\text { and many expressed } \\
\text { that they would stop } \\
\text { buying products with } \\
\text { nutrient warning signs. } \\
\text { There were significant } \\
\text { differences by } \\
\text { nutritional status and } \\
\text { socioeconomic level, } \\
\text { but gender was not a } \\
\text { significant influence. }\end{array}$ \\
\hline
\end{tabular}




\begin{tabular}{|c|c|c|c|c|c|}
\hline Reference & Country & Study design & $\begin{array}{l}\text { Quality } \\
\text { score }\end{array}$ & Objective & Main outcomes \\
\hline $\begin{array}{l}\text { Bezbaruah } \\
\text { and Brunt, } \\
2012 \text { [53] }\end{array}$ & USA & $\begin{array}{l}\text { Quantitative. } \\
\text { Descriptive, } \\
\text { cross- } \\
\text { sectional. }\end{array}$ & LOW & $\begin{array}{l}\text { To determine } \\
\text { the influence of } \\
\text { cartoon } \\
\text { characters in } \\
\text { fruit and } \\
\text { vegetable } \\
\text { preferences } \\
\text { among children } \\
\text { ( } 9-11 \text { years } \\
\text { old }) \text {. }\end{array}$ & $\begin{array}{l}\text { The children reported } \\
\text { that their } \\
\text { fruit/vegetable } \\
\text { preferences were } \\
\text { determined by flavor } \\
\text { and nutritional value. } \\
\text { There were no } \\
\text { significant differences } \\
\text { between genders. }\end{array}$ \\
\hline $\begin{array}{l}\text { Vila-López } \\
\text { and Kuster- } \\
\text { Boluda, } 2016 \\
\text { [54] }\end{array}$ & Spain & $\begin{array}{l}\text { Quantitative. } \\
\text { Descriptive, } \\
\text { cross- } \\
\text { sectional. }\end{array}$ & $\mathrm{HIGH}$ & $\begin{array}{l}\text { To explore } \\
\text { gender } \\
\text { differences in } \\
\text { the association } \\
\text { between } \\
\text { perception of } \\
\text { food packaging } \\
\text { cues and health } \\
\text { motivations } \\
\text { and weight } \\
\text { control in } \\
\text { adolescents } \\
\text { (14-17 years } \\
\text { old). }\end{array}$ & $\begin{array}{l}\text { Girls were more } \\
\text { concerned about } \\
\text { weight control and } \\
\text { health and paid more } \\
\text { attention to informative } \\
\text { cues on food } \\
\text { packaging when } \\
\text { motivated to control } \\
\text { weight, compared to } \\
\text { boys. Visual cues were } \\
\text { not relevant for both } \\
\text { genders. }\end{array}$ \\
\hline $\begin{array}{l}\text { Marquis et } \\
\text { al., } 2005 \text { [55] }\end{array}$ & Canada & $\begin{array}{l}\text { Quantitative. } \\
\text { Descriptive, } \\
\text { cross- } \\
\text { sectional. }\end{array}$ & LOW & $\begin{array}{l}\text { To evaluate the } \\
\text { association } \\
\text { between eating } \\
\text { while watching } \\
\text { TV and food- } \\
\text { related } \\
\text { behaviors in } \\
\text { children } \\
\text { (10 years old). }\end{array}$ & $\begin{array}{l}\text { Eating while watching } \\
\text { TV was found to be } \\
\text { significantly } \\
\text { associated to } \\
\text { unhealthier food } \\
\text { choices. Significant } \\
\text { correlations were found } \\
\text { between the frequency } \\
\text { of eating while } \\
\text { watching TV, the } \\
\text { importance given to a } \\
\text { food's appearance and } \\
\text { children's requests for } \\
\text { advertised foods, but } \\
\text { only among boys. }\end{array}$ \\
\hline
\end{tabular}




\begin{tabular}{|c|c|c|c|c|c|}
\hline Reference & Country & Study design & $\begin{array}{l}\text { Quality } \\
\text { score }\end{array}$ & Objective & Main outcomes \\
\hline $\begin{array}{l}\text { Kaur and } \\
\text { Vohra, } 2013 \\
\text { [56] }\end{array}$ & India & $\begin{array}{l}\text { Quantitative. } \\
\text { Descriptive, } \\
\text { cross- } \\
\text { sectional. }\end{array}$ & MEDIUM & $\begin{array}{l}\text { To analyze the } \\
\text { effectiveness } \\
\text { of in-store food } \\
\text { promotion } \\
\text { strategies } \\
\text { targeting } \\
\text { children. }\end{array}$ & $\begin{array}{l}\text { Free gifts highlighted } \\
\text { on packaging, } \\
\text { assortment of foods, } \\
\text { and placement of } \\
\text { packaged foods in } \\
\text { shelf locations directly } \\
\text { accessible to children } \\
\text { were found to be } \\
\text { among the most } \\
\text { effective in-store } \\
\text { promotional strategies. } \\
\text { Food requests were } \\
\text { more strongly affected } \\
\text { by these influences } \\
\text { among boys than } \\
\text { among girls. }\end{array}$ \\
\hline $\begin{array}{l}\text { Vohra and } \\
\text { Soni, } 2015 \\
\text { [57] }\end{array}$ & India & $\begin{array}{l}\text { Quantitative. } \\
\text { Descriptive, } \\
\text { cross- } \\
\text { sectional. }\end{array}$ & LOW & $\begin{array}{l}\text { To identify the } \\
\text { variables that } \\
\text { predict } \\
\text { shopping } \\
\text { behavior in } \\
\text { retail stores } \\
\text { among } \\
\text { children. }\end{array}$ & $\begin{array}{l}\text { Retail shopping } \\
\text { behavior in children is } \\
\text { influenced by food } \\
\text { promotions in stores, } \\
\text { the frequency with } \\
\text { which the child } \\
\text { accompanies their } \\
\text { mothers to the store, } \\
\text { the age of the parents } \\
\text { and maternal } \\
\text { educational level. } \\
\text { There was no } \\
\text { significant difference } \\
\text { among genders. }\end{array}$ \\
\hline $\begin{array}{l}\text { Busse and } \\
\text { Díaz, } 2016 \\
\text { [58] }\end{array}$ & Peru & $\begin{array}{l}\text { Quali- } \\
\text { quantitative } \\
\text { Descriptive, } \\
\text { cross- } \\
\text { sectional }\end{array}$ & HIGH & $\begin{array}{l}\text { To explore } \\
\text { habits } \\
\text { regarding TV } \\
\text { and food } \\
\text { behaviors } \\
\text { among } \\
\text { Peruvian } \\
\text { children. }\end{array}$ & $\begin{array}{l}\text { Boys and girls reported } \\
\text { different preferences in } \\
\text { TV shows, suggesting } \\
\text { differences in exposure } \\
\text { to food-related TV } \\
\text { content. Some girls } \\
\text { demonstrated } \\
\text { awareness of the } \\
\text { persuasive intent in TV } \\
\text { ads, while others did } \\
\text { not. In contrast, all } \\
\text { boys were aware of the } \\
\text { persuasive intent in } \\
\text { advertising. }\end{array}$ \\
\hline $\begin{array}{l}\text { Bunting et al., } \\
2013 \text { [59] }\end{array}$ & $\begin{array}{l}\text { New } \\
\text { Zealand }\end{array}$ & $\begin{array}{l}\text { Qualitative. } \\
\text { Descriptive, } \\
\text { cross- } \\
\text { sectional }\end{array}$ & MEDIUM & $\begin{array}{l}\text { To evaluate } \\
\text { perceptions } \\
\text { and knowledge } \\
\text { about energy } \\
\text { drinks in three } \\
\text { age groups: } \\
16-21,22-28 \\
\text { and } 29- \\
35 \text { years of age }\end{array}$ & $\begin{array}{l}\text { Participants expressed } \\
\text { awareness regarding } \\
\text { the marketing of } \\
\text { different energy drinks } \\
\text { as predominantly } \\
\text { "feminine" or } \\
\text { "masculine" and the } \\
\text { resulting manipulation } \\
\text { of consumer choices } \\
\text { by the food industry. }\end{array}$ \\
\hline
\end{tabular}




\begin{tabular}{|c|c|c|c|c|c|}
\hline Reference & Country & Study design & $\begin{array}{l}\text { Quality } \\
\text { score }\end{array}$ & Objective & Main outcomes \\
\hline \multirow[t]{2}{*}{$\begin{array}{l}\text { Hattersley et } \\
\text { al., 2009 [60] }\end{array}$} & Australia & $\begin{array}{l}\text { Qualitative. } \\
\text { Descriptive, } \\
\text { cross- } \\
\text { sectional }\end{array}$ & MEDIUM & $\begin{array}{l}\text { To explore } \\
\text { knowledge, } \\
\text { attitudes and } \\
\text { behaviors } \\
\text { related to soft } \\
\text { drink marketing } \\
\text { in university } \\
\text { students (18- } \\
30 \text { years old) }\end{array}$ & $\begin{array}{l}\text { Identified influences on } \\
\text { consumption of soft } \\
\text { drinks were social, } \\
\text { environmental (ready } \\
\text { availability, low prices, } \\
\text { etc), intrinsic qualities } \\
\text { of beverages (taste, } \\
\text { caffeine content, etc.) } \\
\text { and personal health } \\
\text { beliefs. were identified } \\
\text { as important influences } \\
\text { on consumption. }\end{array}$ \\
\hline & & & & & $\begin{array}{l}\text { Men tended to be more } \\
\text { strongly affected by } \\
\text { soft drink marketing } \\
\text { than women. }\end{array}$ \\
\hline $\begin{array}{l}\text { Elliott, } 2009 \\
\text { [61] }\end{array}$ & Canada & $\begin{array}{l}\text { Qualitative. } \\
\text { Descriptive, } \\
\text { cross- } \\
\text { sectional }\end{array}$ & MEDIUM & $\begin{array}{l}\text { To analyze } \\
\text { differences in } \\
\text { attitudes and } \\
\text { responses of } \\
\text { boys and girls } \\
\text { towards "fun } \\
\text { food" } \\
\text { marketing } \\
\text { techniques. }\end{array}$ & $\begin{array}{l}\text { Girls were more likely } \\
\text { to choose products } \\
\text { based on color } \\
\text { preferences and } \\
\text { aesthetic appeal, while } \\
\text { boys were more } \\
\text { interested in the } \\
\text { interactive features of } \\
\text { the products. }\end{array}$ \\
\hline
\end{tabular}


Table 2

Classification of included studies by theme, finding of differences by gender and methodological quality.

\begin{tabular}{|c|c|c|c|c|c|c|}
\hline \multirow{3}{*}{$\begin{array}{l}\text { Theme / } \\
\text { methodological } \\
\text { quality }\end{array}$} & \multicolumn{6}{|c|}{ Gender differences } \\
\hline & \multicolumn{3}{|l|}{ Yes } & \multicolumn{2}{|l|}{ No } & \multirow[b]{2}{*}{ Low } \\
\hline & High & Medium & Low & High & Medium & \\
\hline \multirow{7}{*}{$\begin{array}{l}\text { Effect of food } \\
\text { marketing on } \\
\text { food intake } \\
\text { among children } \\
\text { and } \\
\text { adolescents }\end{array}$} & & & \multicolumn{2}{|l|}{$\begin{array}{l}\cdot \text { Anschutz } \\
\text { et al } \\
2011[35]\end{array}$} & $\begin{array}{l}\text { - Cornwell et } \\
\text { al 2014[44] }\end{array}$ & $\begin{array}{l}\text { - Ueda et al } \\
\text { 2012[23] }\end{array}$ \\
\hline & & & \multirow{2}{*}{\multicolumn{2}{|c|}{$\begin{array}{l}\cdot \text { Anschutz } \\
\text { et al } \\
2009[30]\end{array}$}} & \multirow[t]{6}{*}{$\begin{array}{l}\text { - Norman et } \\
\text { al 2010[26] }\end{array}$} & $\begin{array}{l}\text { - Buijzen et } \\
\text { al 2008[50] }\end{array}$ \\
\hline & & & & & & \multirow{2}{*}{$\begin{array}{l}- \text { Keller et al } \\
2012 \\
\text { (study 1) } \\
{[29]}\end{array}$} \\
\hline & & & \multicolumn{2}{|l|}{$\begin{array}{l}\text { et al } \\
2015[27]\end{array}$} & & \\
\hline & & & \multirow{3}{*}{$\begin{array}{l}\text { - Keller et al } \\
2012 \text { (study } \\
\text { 2)[29] }\end{array}$} & & & $\begin{array}{l}\text { •Anschutz } \\
\text { 2010[39] }\end{array}$ \\
\hline & & & & & & $\begin{array}{l}\text { - Baldwin et } \\
\text { al 2018[46] }\end{array}$ \\
\hline & & & & & & $\begin{array}{l}\text { Wonderlich- } \\
\text { Tierney et } \\
\text { al 2013[34] }\end{array}$ \\
\hline \multirow{3}{*}{$\begin{array}{l}\text { Effect of food } \\
\text { advertising on } \\
\text { preferences } \\
\text { and choice }\end{array}$} & & $\begin{array}{l}\text { •Chernin } \\
\text { 2008[24] }\end{array}$ & \multirow{2}{*}{$\begin{array}{l}\text { Castonguay } \\
\text { \& Bakir } \\
\text { (study 1) } \\
\text { [17] }\end{array}$} & \multirow{3}{*}{$\begin{array}{l}\text { - } \\
\text { Olivares- } \\
\text { Cortés } \\
2017[52]\end{array}$} & $\begin{array}{l}\cdot \\
\text { Tarabashkina } \\
2017[25]\end{array}$ & $\begin{array}{l}\text { - Vohra \& } \\
\text { Soni } 2015 \\
\text { [57] }\end{array}$ \\
\hline & & Vohra & & & - Olivares et & - Velazquez \\
\hline & & & $\begin{array}{l}\text { - Marquis et } \\
\text { al 2005[55] }\end{array}$ & & & $2014[28]$ \\
\hline $\begin{array}{l}\text { Responses to } \\
\text { specific } \\
\text { marketing }\end{array}$ & $\begin{array}{l}\text { - Vila-López } \\
\text { \& Kuster- } \\
\text { Boluda[54] }\end{array}$ & $\begin{array}{l}\text { • Elliott } \\
2009[61]\end{array}$ & $\begin{array}{l}\text { - Ogle et al } \\
2017[33]\end{array}$ & & & $\begin{array}{l}\text { - Adams \& } \\
\text { Geuens } \\
2007[36]\end{array}$ \\
\hline techniques & & $\begin{array}{l}\text { Hattersley } \\
\text { 2009[60] }\end{array}$ & $\begin{array}{l}\text { - Gines } \\
\text { Geraldo \& } \\
\text { Machado } \\
\text { Silva } \\
\text { 2012[38] }\end{array}$ & & & $\begin{array}{l}\text { Bezbaruah } \\
\text { \& Brunt } \\
\text { 2012[53] }\end{array}$ \\
\hline & & & $\begin{array}{l}\text { • Hobin et al } \\
2012[32]\end{array}$ & & & $\begin{array}{l}\text { - Harris et } \\
\text { al 2018[37] }\end{array}$ \\
\hline & & & $\begin{array}{l}\text { - Dixon et al } \\
2014[31]\end{array}$ & & & \\
\hline
\end{tabular}




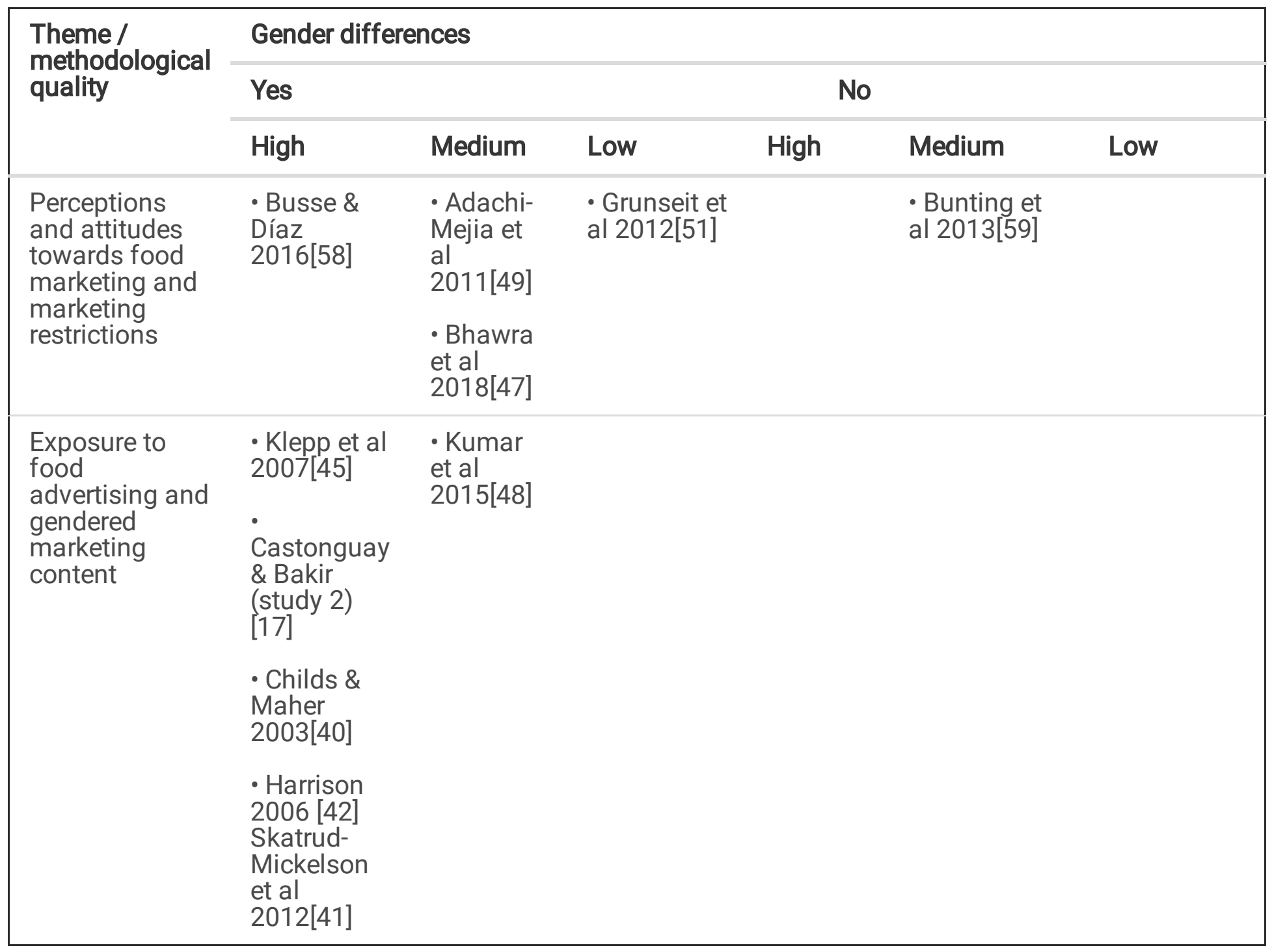

Table 1 presents a summary of each study's main objective and outcomes, as well as its quality score. Table 2 illustrates the distribution of studies of low, medium and high methodological quality within each dimension of interest.

\section{Gender differences in the effect of food marketing on food intake among children and adolescents}

A group of twelve studies analyzed the differential effects of food marketing on eating behaviors among children and adolescents, particularly of exposure to food advertising in different media (TV, internet, etc.), although TV was the most frequently investigated one. Most of these studies were classified as having low methodological quality (Table 2). Only four studies[27, 29, 30, 35] found significant differences between genders, but the gender identified as more strongly influenced by food marketing varied among studies.

An experimental study conducted in the Netherlands on children of 8 to 12 years of age by Anschutz et al. [30] reported that boys tended to eat a greater amount of food when viewing TV shows embedded with food ads vs. non-food ads, but this association was not found among girls. Also in the Netherlands, Anschutz et al. [35] found a higher food intake among young female university students (20 years old on average) 
exposed to food ads, compared to the women exposed to neutral ads. The opposite association was found in young men, who tended to consume less food when exposed to food ads vs. non-food ads.

Finally, a study conducted by Anderson et al. [27] on children and adolescents (9-14 years old) in Canada found that girls with overweight/obesity were more vulnerable to marketing techniques and increased their energy intake after exposure to food ads, while girls with normal weight and boys did not. Similar results were obtained by Keller et al. [29] in the USA to determine the impact of familiar fast-food branding on testmeal intake in children (7-9 years old). Girls were found to increase food intake in the presence of familiar fast-food brands, while boys ate similar amounts when presented with fast-food branded items vs. neutral food items (i.e. in plain white containers).

The eight remaining studies that analyzed the effect of exposure to food advertising did not observe significant differences between genders[23, 26, 29, 34, 39, 44, 46, 50], although they made some noteworthy observations regarding this influence and other intervening variables. One study found that brand recognition in TV ads of products high in sugar, fat and salt was a predictor of $\mathrm{BMI}$ in children as young as 3 years old[44], while other studies observed that maternal encouragement to be thin[39] and intra-family communication[50] had a moderating effect in the impact of food advertising in school-aged children. Young children with overweight were found to be more responsive to branding of food items than children with normal weight[29]. More recent studies have also explored the impact of online advertising. Exposure to online food ads was found to be associated with increased consumption of unhealthy food products[46] and the influence of food advertising was observed to depend on the type of ad and media[26]. Finally, exposure to food ads in interaction with transportability, or the tendency to become engrossed in what one is viewing, was also found to have a significant effect on food intake in undergraduate university students[34].

\section{Gender differences in the effects of advertising on preferences and choice among children and adolescents.}

A group of nine studies explored these issues, of which four $[17,24,55,56]$ generated evidence that food advertising exerts a more powerful influence on choices and preferences among male children and adolescents than among females.

An experimental study by Chernin et al. on children aged 5 to 11 years old in the USA found that exposure to food advertising increased the children's preference for the advertised products, an effect that was more powerful among boys[24]. Also in the USA, Castonguay \& Bakir[17] evaluated attitudes towards maintaining an active lifestyle among school-aged children after exposure to ads portraying physical activities vs. neutral ads. Boys manifested a greater intention to engage in physical activity when exposed to ads depicting sports, compared to girls overall and to boys who only viewed neutral ads. The authors concluded that boys are more likely to believe that the energy-dense, low quality foods depicted in these ads will help boost their physical performance than girls.

In Canada, Marquis et al. [55] evaluated how eating while watching TV affected purchase requests among school-aged children. Boys reported asking their parents to purchase the advertised products more frequently than girls, which suggests that boys were more vulnerable to food advertising than girls. Similar results were obtained by Kaur and Vohra[56] in a cross-sectional study regarding the influence of

Page 21/31 
promotional marketing techniques in retail stores among school-aged children in India. The analysis of questionnaires administered to the children's mothers showed that these promotion strategies affected boys' purchase requests more strongly than girls'.

The remaining six studies that assessed the impact of advertising on preferences and choice did not show significant differences between genders [25, 28, 37, 43, 52, 57]. However, these studies shed light on the specific ways in which this influence operates. These studies did not only confirm advertising and in-store promotional strategies to be powerful influences on food choice and preferences among children and adolescents[57], but in some cases they also identified other factors that moderate this effect, such as nutritional knowledge[25], the degree of attention to advertisements[28] and socioeconomic variables other than gender[52, 57].

\section{Gender differences in responses to specific marketing techniques among children and adolescents.}

Seven[31-33, 38, 54, 60, 61] of the nine studies included in this category found that male and female children and adolescents responded differently to specific marketing techniques.

An experimental study conducted in the USA by Ogle et al. [33] focused on the influence of depictions of licensed cartoon characters in food packaging on the attention and food preferences of school-aged children, finding that children prefer products depicting characters of their same gender. In Australia, Dixon et al.[31] also conducted an experimental study in a similar age group, where male children were observed to be more attracted to products that showed sport celebrities or that included toys, compared to girls. In an experimental study on visual memory among school children in Brazil, Gines Geraldo and Machado Pinto e Silva [38]found that girls could remember images and characters to a greater degree than boys, although no significant differences were found between genders in the capacity to remember colors.

Two studies focused on marketing techniques that involve active interaction with products. Using an experimental design, Hobin et al.[32] found that Canadian children were more likely to choose healthier products if toys were offered as a premium only with healthier foods, but this effect was stronger in boys than in girls. In turn, by means of focal groups, Elliot [61] explored the marketing techniques involved in "fun foods" and observed that products with enhanced interactive features were more appealing to male schoolchildren, whereas female choices were more determined by aesthetic and associative values.

As for adolescents, Vila-López and Kuster-Boluda[54] explored the importance of visual and information cues in food packages in the attitudes and preferences of adolescents in Spain. While visual cues were not found to be a significant influence for either gender, information cues were observed to affect female adolescents more strongly than males, because females tended to be more worried about weight control and health overall and these factors played more heavily on their food choices.

Finally, Hattersley et al. [60] assessed gender differences in responses to the marketing of soft drinks among young Australian adults (18-30 years old). This study found that men were more influenced by the marketing of soft drinks, while women were more strongly influenced by the promotion of "healthier options" (e.g. fruit juice). 
Of three studies that did not observe significant differences between genders, one found that the interaction of healthy/unhealthy slogans with healthy/unhealthy products had a significant influence in purchase intent among 15-year-olds in Belgium [36]. Another study assessed the potential of using cartoon characters to promote fruit and vegetable consumption among children, with varying results by type of character and age of the audience[53]. The third paper corroborated the power of health claims and their impact on children's perceptions of relative healthfulness of food products with low nutritional quality[37].

\section{Gender differences in perceptions and attitudes towards food marketing and marketing regulation initiatives.}

This group includes five studies[47, 49, 51, 58,59] whose findings suggest that children and adolescents presented some level of awareness regarding the persuasive intent behind marketing, particularly gendered advertisements, and that males and females have different perspectives regarding the need for regulation of food marketing.

Focus groups conducted by Bunting et al.[59] in New Zealand explored perceptions involving the marketing of energy drinks among adolescents and young adults (16 to 35 years old). All participants demonstrated being highly aware of the fact that energy drinks advertising targeted preferentially men or women, and that this implies a certain level of manipulation of audiences to choose products based on gendered perceptions. In contrast, a similar design implemented by Busse and Díaz[58] among school-aged Peruvian children found that all boys were aware of the persuasive intent in TV advertising, but some girls did not, suggesting a greater vulnerability to advertising persuasion in girls than in boys.

Adachi-Mejia et al. [49] assessed receptivity to TV advertising among 10 to 13 years old in the USA, in which having a favorite advertisement was used as a measure of receptivity. Although both girls and boys showed similar receptivity levels, among respondents who declared having a food ad as their favorite, boys were more likely to prefer a beer ad than girls. Conversely, girls were more likely to name a candy ad than boys.

Bhawra et al.[47] and Grunseit et al.[51] gauged the support for food marketing regulations among adolescents and young adults (16 to 30 years old) in Canada and young Australian athletes ( 15 to 23 years old), respectively. In both cases, women were more likely to support food marketing restrictions and related policies. Additionally, female athletes also showed a greater level of disagreement with the promotion of unhealthy foods associated to sports than men[51].

\section{Gender differences in exposure to food advertising and gendered marketing content targeting children and adolescents.}

There were six studies[17, 40-42, 45, 48] that explored either gender differences in exposure intensity or gendered marketing content. Results suggest that boys were exposed to food advertising more frequently than girls and that there is a gender bias in food marketing.

When administered questionnaires, boys reported that they viewed/listened to food ads more often and spent more time watching TV than girls, both in the USA[48] and Europe[45]. 
Studies focusing on advertising content found significant differences in both the frequency with which each gender was portrayed and the associated types of messages or foods. Castonguay and Bakir[17] observed that only $18.8 \%$ of TV ads in the USA had exclusively female characters and $6.7 \%$ showed both genders, while $74.6 \%$ showed only males. Moreover, there was an association between food healthfulness and gender, where women were more likely to be associated to healthful products and men to unhealthful ones. In the UK, Childs and Maher[40] also found that TV ads were gender bias towards boys, who were portrayed more often, either in image or as voice-over, than girls. Harrison[42] also included ethnicity in their study of gendered content in TV advertising, dividing ads into two groups: those portraying Black characters and those that did not. They observed that male characters were overrepresented in both groups $(62.7 \%$ and $65.4 \%)$.

Skatrud-Mickelson et al.[41] followed a very different approach to this issue and estimated exposure by combining audience composition data, ticket sales for the top-20 box-office movies in the USA, and the number of times specific food brands appeared on screen in each of these movies. Their results suggest that gender differences in exposure to advertising were determined by the interaction of age and movie age rating. Exposure among girls was greater than boys for ages 6 to 11 and 12 to 17 in G/PG rated movies, while boys of all ages had a higher exposure in PG-13 and R-rated movies.

\section{Discussion}

Children are particularly vulnerable to persuasive messages used in marketing as their cognition is relatively limited for the recognition of marketing communication[62]. Food marketing exposure influences their preferences and, ultimately, their consumption[6,63]. There is already strong evidence that shows the linkage between food marketing and childhood obesity[64]. However, little is known about the role of gender in the promotion of products high in salt, sugar and fat to children and adolescents.

Food products do not have a gender per se, but marketing strategies are constructed in such a way that products have become gendered, resulting in foods that are more appealing to girls or to boys depending on the promotional techniques applied. Most of the studies included in this review agreed that food advertising and promotion has a similar impact on the consumption of unhealthy foods on both boys and girls. Several works found boys to be more frequently exposed to food advertising and their preferences to be more affected by this exposure, as compared to girls. This occurs in a context where advertising content presents gender bias and preferentially targets boys. The studies also bring to light the many variables that could mediate the association among advertising, gender, and eating behaviors, such as weight status, nutritional knowledge, advertising techniques, type of media, brand awareness, intra-family pressures, and others.

\section{Strengths And Weaknesses Of The Selected Studies}

The main strength of this review is that it contributes to the systematization of data regarding the role of gender in food advertising and in providing new research lines for future studies.

One limitation of this study is that the body of research reviewed is heavily biased towards TV advertising, with very few studies focusing on the impact of relatively new marketing channels, such as online games or 
social media platforms. Another limitation is the lack of common definitions for key variables, such as "persuasion" or "attention". It is difficult to compare results, as there is no standardization in the measurement of outcome variables.

Methodological quality was uneven among the studies, although certain dimensions seem to have been explored using consistently more sound methodologies than others, such as gender differences in exposure to advertising and gender bias in advertising content. In contrast, most of the studies assessing the effect of marketing on food intake were of low quality. However, no associations between study quality and the identification of gender differences in any of the issues of interest for this review were identified.

Perhaps the most serious shortcoming of the work discussed in this review is the poor development of a common theoretical framework to investigate gender issues. Most of the studies used biological sex as a grouping variable, taking gender as an unproblematic construct that could be perfectly equated to sex. In order to make a meaningful contribution to gender studies, the research of the influence of food marketing on the eating behaviors of girls and boys must account for the cultural, economic and social factors that come into play in the construction of gender identities and how these factors influence the relationship between food advertising and people, mediated by social expectations and stereotyped "masculine" and "feminine" behaviors, attitudes, and preferences[65]. Most of the studies reviewed here have taken a simplistic approach that limits the question to biological, genetic or metabolic differences between the sexes, which also precludes any possible consideration of other, non-binary gender identities. Moreover, most of the studies were conducted in Europe, the USA or Australia, with little to no representation of the developing world, where gender disparities could be much greater than in these high-income Western countries.

\section{Areas identified for future research}

The body of work explored in this scoping review is a testimony to the increasing interest of the academic community in exploring gender-related determinants to eating behaviors and its implications for disparities in public health. We have identified issues that would particularly benefit from further inquiry, especially in the developing world, including gender stereotypes portrayed and reinforced in food advertising and how they have changed over time, how exposure to advertising varies between children and adults, the impact of new media and the role of influencers.

\section{Conclusions}

This narrative review revealed that gender plays an important role in the development of food marketing techniques and how children respond to them. Boys and girls react in a different way to marketing, and some studies have shown that boys could be more affected, not only because they are more intensively exposed to food advertising, but also because food advertisements are usually male-oriented. Male dominance in food advertising targeting children might be a reflection of an existing cultural bias rather than an explicit decision made by food companies, but the issue merits further research, with particular attention to potential gender biases in different food categories, age sub-groups and socio-economic levels. This review also highlights the need to consider confounding variables that could potentially interact with gender 
and explain differential effects of advertising on food intake, including nutritional status, nutritional knowledge, type of marketing strategy and media, and previous contact or familiarity with the advertised brand, among others.

In view of the significance of the gender dimension in food marketing demonstrated in this review, it is imperious to include a gender perspective in policies that aim to regulate food marketing to children and adolescents. Gender is a transversal dimension that interacts and enhances all other forms of health disparities, and it must be considered in policies that address obesogenic environments.

\section{Declarations}

Ethics approval and consent to participate Not applicable.

Consent for publication Not applicable.

Availability of data and materials Not applicable.

Competing interests The authors declare that they have no competing interests.

Funding International Development Research Centre (IDRC) under grant 108645. The funding agency had not role in the design of the study, collection of data, interpretation of results or writing of the manuscript.

Authors' contributions L.C was responsible for the research design, data collection, interpretation of results and writing of the manuscript, L.G. contributed to the research design, data collection and interpretation of results. M.V.T. contributed to the design of the overall research strategy and the interpretation of results. L.A. contributed to the interpretation of results, writing of the manuscript and supervised all the phases of this study. All co-authors reviewed drafts of the paper and contributed to the final manuscript.

Acknowledgements The authors thank Belén Beltramo for her assistance in collecting and analyzing the articles included in this review, Malena Pirola for her assistance in writing the manuscript, and the rest of FIC-Argentina's team for their contribution during the conduct of this study. They also thank the other research teams involved in the collaborative project for their feedback: Joaquín Barnoya; Peter Busse; Lucila Rozas; Sophia Mus, Alejandra Karina Garron Monje, Christiam Arce Vargas.

\section{References}

1. United Nations Children's Fund (UNICEF), World Health Organization, International Bank for Reconstruction Development/The World Bank: Levels and Trends in Child Malnutrition: Key Findings of the 2020 Edition of the Joint Child Malnutrition Estimates. Geneva: World Health Organization; 2020.

2. NCD Risk Factor Collaboration (NCD-RisC): Worldwide trends in children's and adolescents' body mass index, underweight and obesity, in comparison with adults, from 1975 to 2016: a pooled analysis of 2,416 population-based measurement studies with 128.9 million participants. The Lancet 2017, 390:2627-2642. 
3. Pizzi MA, Vroman K: Childhood obesity: effects on children's participation, mental health, and psychosocial development.Occup Ther Health Care 2013, 27:99-112.

4. Pulgarón ER: Childhood Obesity: A Review of Increased Risk for Physical and Psychological Comorbidities.Clin Ther 2013, 35:A18-A32.

5. Boyland EJ, Whalen R: Food advertising to children and its effects on diet: review of recent prevalence and impact data.Pediatr Diabetes 2015, 16:331-337.

6. Boyland EJ, Nolan S, Kelly B, Tudur-Smith C, Jones A, Halford JC, Robinson E: Advertising as a cue to consume: a systematic review and meta-analysis of the effects of acute exposure to unhealthy food and nonalcoholic beverage advertising on intake in children and adults1,2.Am J Clin Nutr 2016, 103:519-533.

7. World Health Organization. Set of recommendations on the marketing of foods and non-alcoholic beverages to children. Geneva2010.

8. Vargas CM, Stines EM, Granado HS: Health-equity issues related to childhood obesity: A scoping review.J Public Health Dent 2017, 77:S32-S42.

9. Corvalán C, Garmendia M, Jones-Smith J, Lutter C, Miranda J, Pedraza L, Popkin B, Ramirez-Zea M, Salvo D, Stein A: Nutrition status of children in Latin America.Obes Rev 2017, 18:7-18.

10. Chemas-Velez MM, Gómez LF, Velasquez A, Mora-Plazas M, Parra DC: Scoping review of studies on food marketing in Latin America: Summary of existing evidence and research gaps.Rev Saude Publica 2019, 53:107.

11. Allemandi L, Castronuovo L, Tiscornia MV, Ponce M, Schoj V: Food advertising on Argentinean television: are ultra-processed foods in the lead? Public Health Nutr 2018, 21:238-246.

12. Kelly B, Vandevijvere S, Ng S, Adams J, Allemandi L, Bahena-Espina L, Barquera S, Boyland E, Calleja P, Carmona-Garcés IC: Global benchmarking of children's exposure to television advertising of unhealthy foods and beverages across 22 countries. Obes Rev 2019, 20:116-128.

13. Pan American Health Organization. Recommendations from a PAHO Expert Consultation on the Marketing of Food and Non-Alcoholic Beverages to Children in the Americas. Washington DC:PAHO, 2011.

14. Letona P, Chacon V, Roberto C, Barnoya J: Effects of licensed characters on children's taste and snack preferences in Guatemala, a low/middle income country.Int J Obes 2014, 38:1466.

15. Cairns G, Angus K, Hastings G, Caraher M: Systematic reviews of the evidence on the nature, extent and effects of food marketing to children. A retrospective summary.Appetite 2013, 62:209-215.

16. Sweeting HN: Gendered dimensions of obesity in childhood and adolescence.Nutr J 2008, 7:1.

17. Castonguay J, Bakir A: You eat "like a girl": gender differences in content and effects of food advertising depicting sports.Food Prod Mark 2019, 25:233-256.

18. Uray N, Burnaz S: An analysis of the portrayal of gender roles in Turkish television advertisements.Sex Roles 2003, 48:77-87.

19. Royo-Vela M, Aldas-Manzano J, Küster I, Vila N: Adaptation of marketing activities to cultural and social context: Gender role portrayals and sexism in Spanish commercials.Sex Roles 2008, 58:379-390. 
20. Gentry J, Harrison R: Is advertising a barrier to male movement toward gender change?Marketing Theory 2010, 10:74-96.

21. Levac D, Colquhoun H, O'Brien KK: Scoping studies: advancing the methodology.Implementation Science 2010, 5:69.

22. Leamy M, Bird V, Le Boutillier C, Williams J, Slade M: Conceptual framework for personal recovery in mental health: systematic review and narrative synthesis. Br J Psychiatry 2011, 199:445-452.

23. Ueda P, Tong L, Viedma C, Chandy SJ, Marrone G, Simon A, Lundborg CS: Food marketing towards children: brand logo recognition, food-related behavior and BMI among 3-13-year-olds in a south Indian town.PLoS One 2012, 7:e47000.

24. Chernin A: The effects of food marketing on children's preferences: testing the moderating roles of age and gender.Ann Am Acad Pol Soc Sci 2008, 615:101-118.

25. Tarabashkina L, Quester P, Crouch R: Food advertising, children's food choices and obesity: Interplay of cognitive defences and product evaluation: An experimental study.Int J Obes 2016, 40:581-586.

26. Norman J, Kelly B, McMahon A-T, Boyland E, Baur LA, Chapman K, King L, Hughes C, Bauman A: Sustained impact of energy-dense TV and online food advertising on children's dietary intake: a withinsubject, randomised, crossover, counter-balanced trial.Int J Behav Nutr Phys Act 2018, 15:37.

27. Anderson GH, Khodabandeh S, Patel B, Luhovyy BL, Bellissimo N, Mollard RC: Mealtime exposure to food advertisements while watching television increases food intake in overweight and obese girls but has a paradoxical effect in boys.Appl Physiol Nutr Metab 2015, 40:162-167.

28. Velazquez CE, Pasch KE: Attention to food and beverage advertisements as measured by eye-tracking technology and the food preferences and choices of youth.J Acad Nutr Diet 2014, 114:578-582.

29. Keller KL, Kuilema LG, Lee N, Yoon J, Mascaro B, Combes A-L, Deutsch B, Sorte K, Halford JC: The impact of food branding on children's eating behavior and obesity.Physiol Behav 2012, 106:379-386.

30. Anschutz DJ, Engels RC, Van Strien T: Side effects of television food commercials on concurrent nonadvertised sweet snack food intakes in young children.Am J Clin Nutr 2009, 89:1328-1333.

31. Dixon H, Scully M, Niven P, Kelly B, Chapman K, Donovan R, Martin J, Baur LA, Crawford D, Wakefield M: Effects of nutrient content claims, sports celebrity endorsements and premium offers on pre-adolescent children's food preferences: experimental research.Pediatr Obes 2014, 9:e47-e57.

32. Hobin EP, Hammond DG, Daniel S, Hanning RM, Manske SR: The Happy Meal® effect: the impact of toy premiums on healthy eating among children in Ontario, Canada.Can J Public Health 2012, 103:e244e248.

33. Ogle AD, Graham DJ, Lucas-Thompson RG, Roberto CA: Influence of cartoon media characters on children's attention to and preference for food and beverage products.J Acad Nutr Diet 2017, 117:265270. e262.

34. Wonderlich-Tierney AL, Wenzel KR, Vander Wal JS, Wang-Hall J: Food-related advertisements and food intake among adult men and women.Appetite 2013, 71:57-62.

35. Anschutz DJ, Engels RC, van der Zwaluw CS, Van Strien T: Sex differences in young adults' snack food intake after food commercial exposure.Appetite 2011, 56:255-260. 
36. Adams L, Geuens M: Healthy or unhealthy slogans: That's the question....J Health Commun 2007, 12:173-185.

37. Harris J, Haraghey K, Lodolce M, Semenza N: Teaching children about good health? Halo effects in child-directed advertisements for unhealthy food.Pediatr Obes 2018, 13:256-264.

38. Gines Geraldo AP, Machado Pinto e Silva ME: Processed foods in infant feeding: analysis of the visual memory of schoolchildren in Taubaté city, São Paulo.Journal of Human Growth and Development 2012, 22:53-59.

39. Anschutz DJ, Engels RC, Van Strien T: Maternal encouragement to be thin moderates the effect of commercials on children's snack food intake.Appetite 2010, 55:117-123.

40. Childs NM, Maher JK: Gender in food advertising to children: boys eat first.Br Food J 2003. 105: 408419

41. Skatrud-Mickelson M, Adachi-Mejia AM, MacKenzie TA, Sutherland LA: Giving the wrong impression: food and beverage brand impressions delivered to youth through popular movies.J Public Health 2012, 34:245-252.

42. Harrison K: Fast and sweet: Nutritional attributes of television food advertisements with and without Black characters. The Howard Journal of Communications 2006, 17:249-264.

43. Olivares S, Lera L, Mardones MA, Araneda J, Bustos N, Olivares MA, Colque ME: Promoción de alimentos y preferencias alimentarias en escolares chilenos de diferente nivel socioeconómico.Arch Latinoam Nutr 2011, 61:163.

44. Cornwell TB, McAlister AR, Polmear-Swendris N: Children's knowledge of packaged and fast food brands and their BMI. Why the relationship matters for policy makers.Appetite 2014, 81:277-283.

45. Klepp K-I, Wind M, De Bourdeaudhuij I, Rodrigo CP, Due P, Bjelland M, Brug J: Television viewing and exposure to food-related commercials among European school children, associations with fruit and vegetable intake: a cross sectional study.Int J Behav Nutr Phys Act 2007, 4:46.

46. Baldwin HJ, Freeman B, Kelly B: Like and share: associations between social media engagement and dietary choices in children.Public Health Nutr 2018, 21:3210-3215.

47. Bhawra J, Reid JL, White CM, Vanderlee L, Raine K, Hammond D: Are young Canadians supportive of proposed nutrition policies and regulations? An overview of policy support and the impact of sociodemographic factors on public opinion.Can J Public Health 2018, 109:498-505.

48. Kumar G, Onufrak S, Zytnick D, Kingsley B, Park S: Self-reported advertising exposure to sugarsweetened beverages among US youth.Public Health Nutr 2015, 18:1173-1179.

49. Adachi-Mejia AM, Sutherland LA, Longacre MR, Beach ML, Titus-Ernstoff L, Gibson JJ, Dalton MA: Adolescent weight status and receptivity to food TV advertisements.J Nutr Educ Behav 2011, 43:441448.

50. Buijzen M, Schuurman J, Bomhof E: Associations between children's television advertising exposure and their food consumption patterns: A household diary-survey study.Appetite 2008, 50:231-239.

51. Grunseit AC, MacNiven R, Orr R, Grassmayr M, Kelly B, Davies D, Colagiuri S, Bauman AE: Australian athletes' health behaviours and perceptions of role modelling and marketing of unhealthy products. Health Promot J Austr 2012, 23:63-69. 
52. Olivares-Cortés S, Araneda-Flores J, Morales-Illanes G, Leyton-Dinamarca B, Bustos-Zapata N, Hernández-Moreno M-A, Oyarzún-Macchiavello M-T: Actitudes de escolares chilenos de distinto nivel socioeconómico al inicio de la implementación de la ley que regula la venta y publicidad de alimentos altos en nutrientes críticos. Nutr Hosp 2017, 34:431-438.

53. Bezbaruah $N$, Brunt $A$ : The influence of cartoon character advertising on fruit and vegetable preferences of 9-to 11-year-old children.J Nutr Educ Behav 2012, 44:438-441.

54. Vila-López N, Kuster-Boluda I: Adolescents' food packaging perceptions. Does gender matter when weight control and health motivations are considered? Food Qual Prefer 2016, 52:179-187.

55. Marquis M, Filion YP, Dagenais F: Does eating while watching television influence children's food-related behaviours? Can J Diet Pract Res 2005, 66:12-18.

56. Kaur P, Vohra J: Preference for promotional strategies directed at children: an empirical investigation.Asia Pacific Bus Rev 2013, 5:32-40.

57. Vohra J, Soni P: Logit modelling of food shopping behaviour of children in retail stores.Manag Res Rev 2015, 38:840-854.

58. Busse P, Díaz R: What are the television viewing and eating habits of children in Peru? Glob Health Promot 2016, 23:50-60.

59. Bunting H, Baggett A, Grigor J: Adolescent and young adult perceptions of caffeinated energy drinks. A qualitative approach.Appetite 2013, 65:132-138.

60. Hattersley L, Irwin M, King L, Allman-Farinelli M: Determinants and patterns of soft drink consumption in young adults: a qualitative analysis. Public Health Nutr 2009, 12:1816-1822.

61. Elliott C: Healthy food looks serious: How children interpret packaged food products. Can J Commun 2009, 34: 359-380.

62. Rozendaal E, Buijzen M, Valkenburg P: Comparing children's and adults' cognitive advertising competences in the Netherlands.J Child Media 2010, 4:77-89.

63. World Health Organization. Report of the Commission on Ending Childhood Obesity. 2016. Geneva: World Health Organization.

64. Smith R, Kelly B, Yeatman H, Boyland E: Food marketing influences children's attitudes, preferences and consumption: A systematic critical review.Nutrients 2019, 11:875.

65. García Oliva M: El bueno de Adán y la malvada de Eva. Proceso de enculturación, establecimiento de roles y violencia de género. Ser hombre y ser mujer en Oaxaca. In Mujeres (in) visibles: Género, alimentación y salud en comunidades rurales de Oaxaca.Volume 10. Edited by Arnaiz MG, Pérez Gil Romo SE. Tarragona: Publicacions URV; 2014: 137-171

\section{Figures}




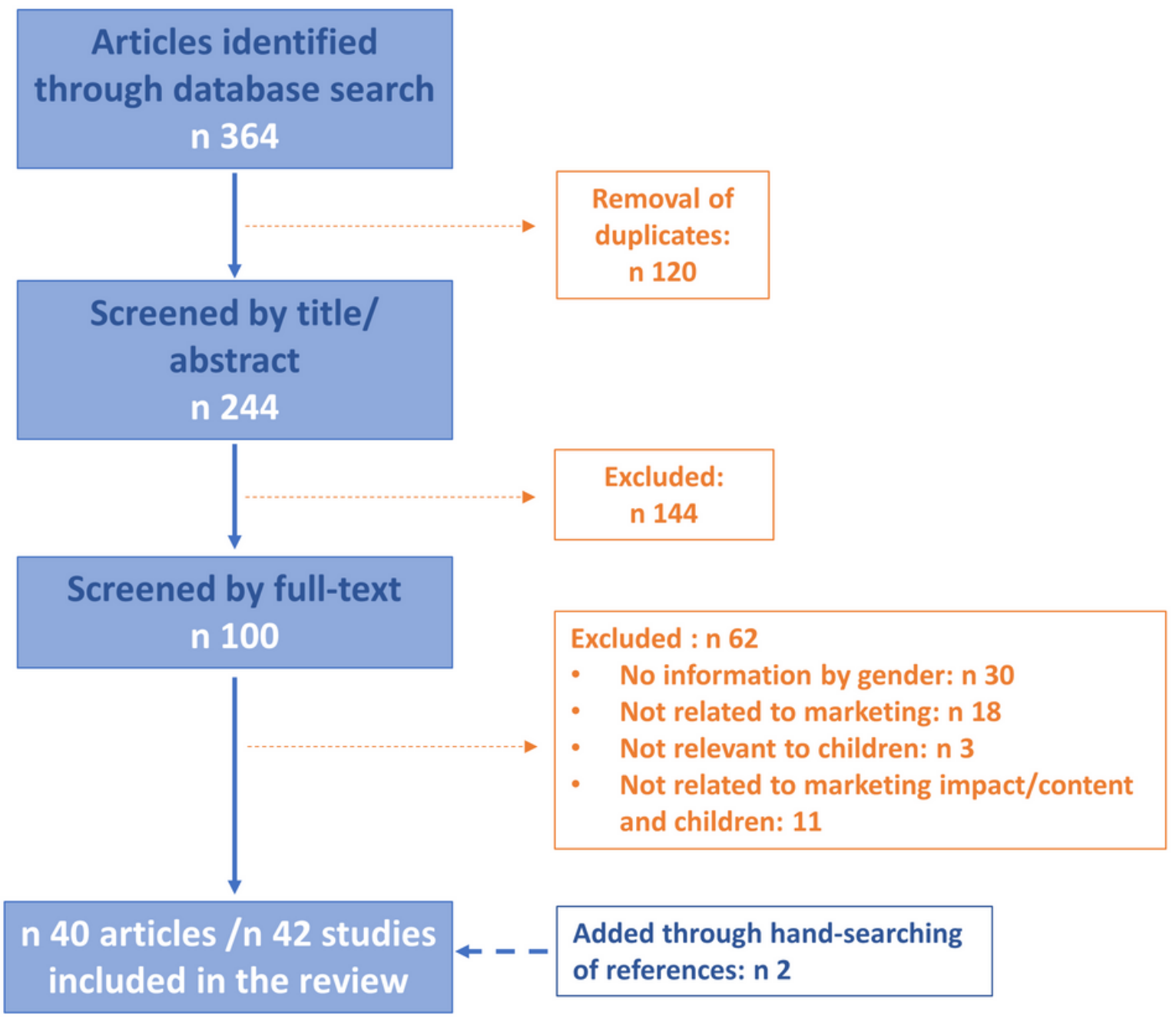

Figure 1

Flow chart of the selection of studies for inclusion in the review. 